\title{
Induction of Apoptosis Scutellaria baicalensis Georgi Root Extract by Inactivation of the Phosphatidyl Inositol 3-kinase/Akt Signaling Pathway in Human Leukemia U937 Cells
}

\author{
Eun Ok Choi ${ }^{1,2}$, Hye-Jin Hwang ${ }^{2,3}$, Yung Hyun Choi ${ }^{1,2}$ \\ ${ }^{1}$ Department of Biochemistry, Dong-Eui University College of Korean Medicine, ${ }^{2}$ Anti-Aging Research Center, Dong-Eui University, ${ }^{3}$ Department \\ of Food and Nutrition, College of Nursing, Healthcare Sciences \& Human Ecology, Dong-Eui University, Busan, Korea
}

Background: The roots of Scutellaria baicalensis Georgi (Labiatae) have been widely used in traditional medicine for treatment of various diseases. In this study, we investigated the effects of ethanol extracts of $S$. baicalensis roots (EESB) on the growth ofn human leukemia U937 cells.

Methods: The effect of EESB on cell viability was measured by the 3-(4,5-dimethyl-2-thiazolyl)-2,5-diphenyl-2H-tetrazolium bromide assay. Apoptosis was determined using 4,6-diamidino-2-phenyllindile staining and flow cytometry. The effects of EESB on the expression of regulatory proteins of apoptosis and phosphatidyl inositol 3-kinase (PI3K)/Akt signaling were determined by Western blotting. Caspase activity and mitochondrial membrane potential (MMP) were measured using flow cytometric analysis.

Results: EESB significantly inhibited the growth of U937 cells and induced apoptosis, which was associated with down-regulation of anti-apoptotic Bcl-2, up-regulation of pro-apoptotic Bax, the loss of MMP and activation of caspase-9 and -3. We also found that EESB enhanced the expression of death receptors (DRs) and their associated ligands and induced the activation of caspase- 8 and truncation of Bid. In addition, EESB suppressed PI3K/Akt signaling and EESB-induced apoptosis and growth inhibition were further increased by inhibition of PI3K activity.

Conclusions: Our results indicated that the pro-apoptotic effect of EESB was mediated through the activation of DR-mediated intrinsic and mitochondria-mediated extrinsic apoptosis pathways and inhibition of the PI3K/Akt signaling in U937 cells.

(J Cancer Prev 2019;24:11-19)

Key Words: Scutellaria baicalensis, U937 cells, Apoptosis, Caspase, PI3K/Akt

\section{INTRODUCTION}

Apoptosis is regulated finely at the genetic level and serves to remove damaged cells during development [1-3]. It is therefore important to identify the apoptotic mechanism for the prevention and treatment of a variety of intractable diseases, including cancer [4-6]. Apoptosis can generally be induced through extrinsic or intrinsic pathways associated with caspase activation $[7,8]$. The extrinsic pathway is activated by stimulation of death receptors (DR) and their ligands in the cell membrane. On the other hand, the intrinsic pathway is initiated through the release of apoptotic factors into the cytoplasm from mitochondria. Both pathways involve the activation of caspases, a class of cysteine proteases $[9,10]$. Therefore, the induction of apoptosis is a critical mechanism for many drug-induced anticancer activities. Induction of apoptosis also involves a variety of intracellular signaling pathways [2,11,12]. Among them, the phosphatidylinositol 3-kinase (PI3K)/Akt pathway is important as a target for cancer therapy because it plays an important role in cell proliferation [9,13]. Activation of PI3K/Akt signaling inhibits expression of pro-apoptotic proteins and enhances cell survival by activating anti-apoptotic proteins

Received February 26, 2019, Revised March 18, 2019, Accepted March 18, 2019

Correspondence to: Yung Hyun Choi

E-mail: choiyh@deu.ac.kr, ORCID: Yung Hyun Choi, https://orcid.org/0000-0002-1454-3124

Copyright (C) 2019 Korean Society of Cancer Prevention

(c) This is an Open Access article distributed under the terms of the Creative Commons Attribution Non-Commercial License (http://creativecommons.org/icenses/by-nc/4.0) which permits unrestricted non-commercial use, distribution, and reproduction in any medium, provided the original work is properly cited. 
[14,15]. Thus, the use of a PI3K inhibitor can completely inhibit Akt activation thereby preventing cancer cell proliferation and inducing programmed cell death.

The roots of Scutellaria baicalensis Georgi (Labiatae) are widely used in traditional Oriental medicine for treating various diseases $[16,17]$. Many recent studies on $S$. baicalensis show a variety of therapeutic effects such as anti-angiogenesis, anti-inflammatory, anti-microbial, immunoenhancing and antioxidative, properties [18-22]. Moreover, several studies have shown that the extracts of $S$. baicalensis roots exhibit various anticancer activities including induction of cell cycle arrest and apoptosis in various cancer cells $[23,24]$. However, the anti-cancer mechanism by $S$. baicalensis in human leukemia cells is not fully understood. In the current study, we investigated the effect of ethanol extract of $S$. baicalensis roots (EESB) on the apoptosis induction of human leukemic U937 cells in relation to the PI3K/Akt pathway.

\section{MATERIALS AND METHODS}

\section{Chemicals and antibodies}

RPMI1640 medium, Dulbecco's modified Eagle's medium (DMEM), FBS, and antibiotics were purchased from WeLGENE Inc. (Daegu, Korea). 4,6-Diamidino-2-phenylindole (DAPI) and MTT were purchased from Sigma-Aldrich Chemical Co. (St. Louis, MO, USA). 5,5',6,6'-Tetrachloro-1,1'3,3'-tetraethyl-imidacarbocyanineiodide (JC-1) and fluorescein-conjugated annexin V (annexin V-FITC) were obtained from Calbiochem (San Diego, CA, USA) and BD Biosciences Pharmingen (San Jose, CA, USA), respectively. The enhanced chemiluminescence (ECL) detection system and in vitro caspase colorimetric assay kit were purchased from Amersham Corp. (Arlington Heights, IL, USA) and R\&D Systems (Minneapolis, MN, USA), respectively. The primary antibodies (Table 1) used for this study were purchased from Bioworld Technology, Inc. (St. Louis Park, MN, USA), Santa Cruz Biotechnology, Inc. (Santa Cruz, CA, USA) and Cell Signaling Technology, Inc. (Beverly, MA, USA). Peroxidase-labeled donkey ant-rabbit and sheep anti-mouse immunoglobulin were purchased from Amersham Corp. All other chemicals not specifically cited here were purchased from Sigma-Aldrich Chemical Co.

\section{Preparation of ethanol extracts of $S$. baicalensis roots}

Roots of $S$. baicalensis were obtained from Dong-Eui Korean Hospital of the Dong-Eui University (Busan, Korea). The roots (40 g) were pulverized into fine powder and immersed in $500 \mathrm{~mL}$ of $70 \%$ ethanol for 2 days. The extracted liquid was filtered twice through Whatman filter paper (No. 3) to remove insoluble matters and concentrated using a rotary evaporator. The extracts (EESB) were redissolved in dimethyl sulfoxide (DMSO) and then diluted with the culture medium to the desired concentration before use. The final DMSO concentration was below the non-cytotoxic range of $0.05 \%$ in all experiments.

Table 1. Antibodies used in the present study

\begin{tabular}{|c|c|c|c|}
\hline Antibody & Dilution & Product No. & Species of origin and supplier \\
\hline Actin & $1: 50,000$ & BS6007M & Mouse polyclonal, Bioworld Technology, Inc. \\
\hline Caspase-3 & $1: 1,000$ & sc-7272 & Mouse polyclonal, Santa Cruz Biotechnology, Inc. \\
\hline Caspase-8 & $1: 1,000$ & sc-7890 & Rabbit polyclonal, Santa Cruz Biotechnology, Inc. \\
\hline Caspase-9 & $1: 1,000$ & sc-7885 & Rabbit polyclonal, Santa Cruz Biotechnology, Inc. \\
\hline PARP & $1: 1,000$ & sc-7150 & Rabbit polyclonal, Santa Cruz Biotechnology, Inc. \\
\hline XIAP & $1: 1,000$ & sc-11426 & Rabbit polyclonal, Santa Cruz Biotechnology, Inc. \\
\hline CIAP-1 & $1: 1,000$ & sc-7943 & Rabbit polyclonal, Santa Cruz Biotechnology, Inc. \\
\hline TRAIL & $1: 1,000$ & sc-7877 & Rabbit polyclonal, Santa Cruz Biotechnology, Inc. \\
\hline DR4 & $1: 1,000$ & sc-7863 & Rabbit polyclonal, Santa Cruz Biotechnology, Inc. \\
\hline DR5 & $1: 1,000$ & sc-65314 & Mouse polyclonal, Santa Cruz Biotechnology, Inc. \\
\hline Fas & $1: 1,000$ & sc-715 & Rabbit polyclonal, Santa Cruz Biotechnology, Inc. \\
\hline FasL & $1: 1,000$ & sc-957 & Rabbit polyclonal, Santa Cruz Biotechnology, Inc. \\
\hline Bcl-2 & $1: 1,000$ & sc-783 & Rabbit polyclonal, Santa Cruz Biotechnology, Inc. \\
\hline Bax & $1: 1,000$ & sc-493 & Rabbit polyclonal, Santa Cruz Biotechnology, Inc. \\
\hline Bid & $1: 1,000$ & sc-11423 & Rabbit polyclonal, Santa Cruz Biotechnology, Inc. \\
\hline PI3K & $1: 1,000$ & $4257 \mathrm{P}$ & Rabbit polyclonal, Cell Signaling Technology, Inc. \\
\hline $\mathrm{p}$-PI3K & $1: 1,000$ & $4228 \mathrm{P}$ & Rabbit polyclonal, Cell Signaling Technology, Inc. \\
\hline Akt & $1: 1,000$ & sc-8312 & Rabbit polyclonal, Santa Cruz Biotechnology, Inc. \\
\hline p-Akt & $1: 1,000$ & sc-101629 & Rabbit polyclonal, Santa Cruz Biotechnology, Inc. \\
\hline
\end{tabular}

IAP, inhibitor of apoptosis protein; DR, death receptor; PI3K, phosphatidyl inositol 3-kinase. 


\section{Cell culture}

The six cancer cell lines used in this study were obtained from American Type Culture Collection (Manassas, MD, USA). Among them, U937 human leukemia and A549 lung carcinoma cells were maintained in RPMI 1640 medium containing $10 \% \mathrm{FBS}, 100 \mu \mathrm{g} / \mathrm{mL}$ streptomycin and $100 \mathrm{U} / \mathrm{mL}$ penicillin in a humidifying incubator containing $5 \% \mathrm{CO}_{2}$ at $37^{\circ} \mathrm{C}$. Hep3B hepatoma, B16F10 mouse melanoma, MDA-MB-231 and MCF-7 breast carcinoma cells were maintained in DMEM supplemented with 10\% FBS and antibiotics at the same condition.

\section{MTT assay}

In brief, after treatment with various concentrations of EESB for 24 hours, $0.5 \mathrm{mg} / \mathrm{mL}$ of MTT solution was added to the culture plates, and they were incubated for 3 hours at $37^{\circ} \mathrm{C}$ and then DMSO was added to dissolve the formazan crystals. The absorbances were measured using an ELISA reader (Molecular Devices, Sunnyvale, CA, USA) at $540 \mathrm{~nm}$.

\section{4,6-Diamidino-2-phenylindole staining}

Cells treated with or without EESB were washed with PBS and fixed with $4 \%$ paraformaldehyde for 10 minutes at room temperature (RT). The cells were washed with PBS and stained with DAPI solution $(2.5 \mu \mathrm{g} / \mathrm{mL})$ for 10 minutes at RT. The cells were then washed twice with PBS and observed with fluorescence microscopy (Carl Zeiss, Deisenhofen, Germany).

\section{Annexin V-FITC staining}

For quantitative assessment of induction of apoptosis rate, the annexin V-FITC staining assay was performed according to the manufacturer's protocol. Briefly, the cells were stained with annexin V-FITC and propidium iodide. After incubation for 15 minutes at RT in the dark, the degree of apoptosis was quantified using flow cytometry (FACS Caliber; Becton Dickinson, San Jose, CA, USA) as the percentage of annexin V-positive cells.

\section{Protein extraction and Western blot analysis}

The cells were lysed for 30 minutes at $4^{\circ} \mathrm{C}$ as previously
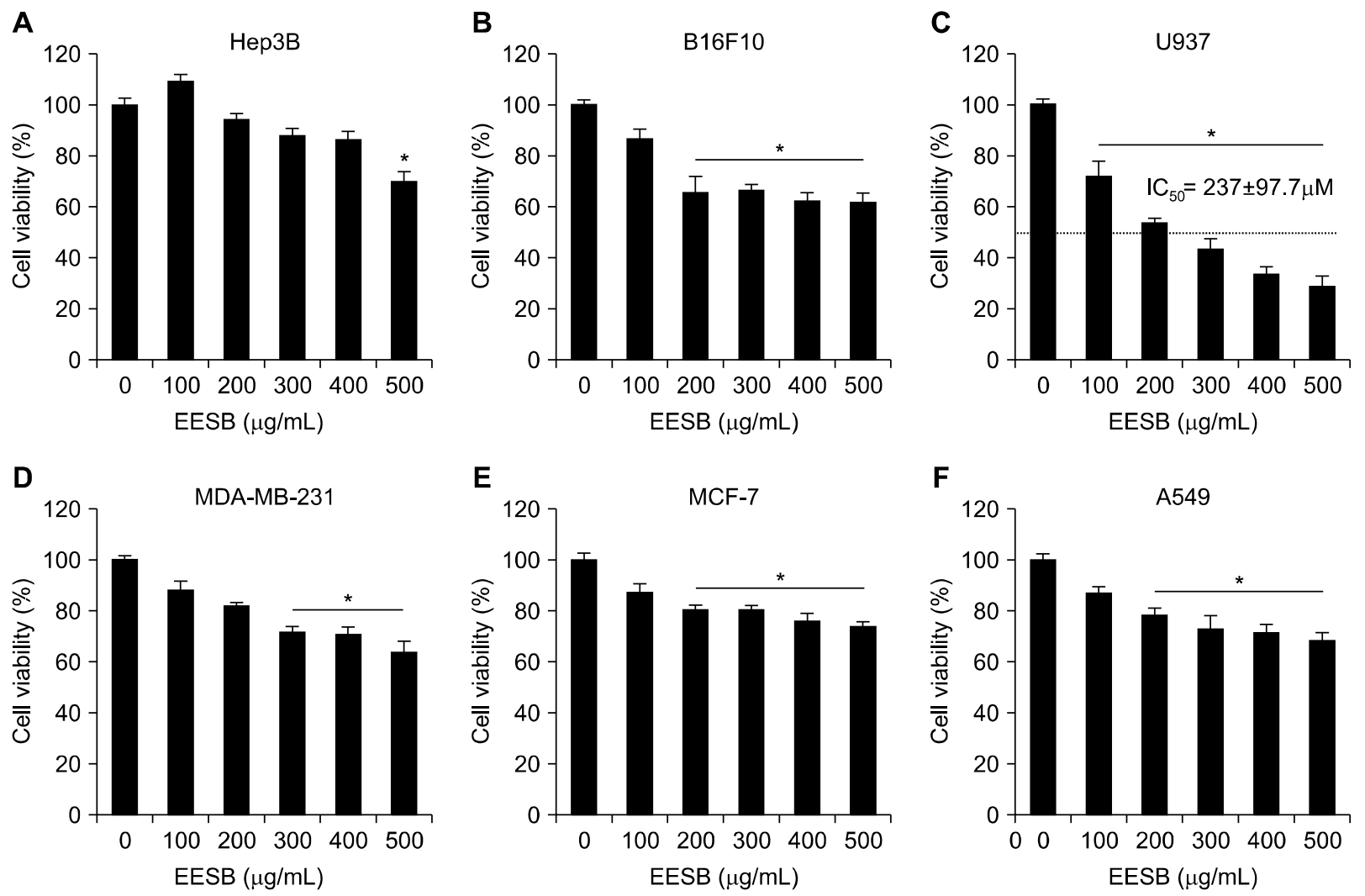

Figure 1. Effects of ethanol extracts of Scutellaria baicalensis roots (EESB) on cell viability in various cancer cell lines. Hep3B hepatoma (A), B16F10 melanoma (B), U937 leukemia (C), MDA-MB-231 and MCF-7 breast carcinoma (D, E), and A549 lung carcinoma (F) cells were treated with the indicated concentrations of EESB for 24 hours. The cell viabilities were determined by the MTT assay. Data are presented as mean \pm SD values obtained from three independent experiments. $* P<0.05$ compared with the untreated control group. 
described [21]. The protein concentration in supernatants was determined using a Bio-Rad protein assay kit (Bio-Rad Laboratories, Hercules, CA, USA) according to the manufacturer's instructions. Equal amounts of protein extract were separated by denaturing SDS PAGE and transferred to polyvinylidene fluoride membranes (Schleicher \& Schuell, Keene, NH, USA). The membranes were incubated overnight at $4^{\circ} \mathrm{C}$ with primary antibodies (Table 1), probed with enzyme-linked secondary antibodies for 1 hour at RT, and detected using an ECL detection system.

\section{Caspase activity assay}

The activities of caspases were determined using colorimetric assay kits, which utilize synthetic tetrapeptides labeled with p-nitroaniline. Briefly, the cells were lysed in the supplied lysis buffer and then the supernatants were incubated with the supplied reaction buffer containing DTT and Asp-Glu-Val-Asp [DEAD] for caspase-3, Ile-Glu-Thr-Asp [IETD] for caspase-8, and Leu-Glu-His-Asp [LEHD] for caspase- 9 as substrates at $37^{\circ} \mathrm{C}$ for 2 hours in the dark. The reactions were measured by changes in absorbance at $405 \mathrm{~nm}$ using an ELISA reader.

\section{Measurement of mitochondrial membrane potential}

The values of mitochondrial membrane potential (MMP) $(\Delta \psi \mathrm{m})$ of cells were determined using JC-1 staining. Briefly, the cells were collected and incubated with $10 \mu \mathrm{M}$ of JC-1 for 20 minutes at $37^{\circ} \mathrm{C}$ in the dark. After removing JC-1, the cells were washed with PBS to remove unbound dye, and the amount of JC-1 retained was measured using flow cytometry.

\section{Statistical analysis}

All experiments were performed at least three times. Data were analyzed using GraphPad Prism software ver. 5.03 (GraphPad Software, Inc., La Jolla, CA, USA), and expressed as the mean \pm SD. Differences between groups were assessed using analysis of variance followed by ANOVA-Tukey's post hoc test, and $P<0.05$ was considered to indicate a statistically significant difference.

\section{RESULTS}

\section{Ethanol extracts of $S$. baicalensis roots inhibit cell viability in various cancer cell lines}

To examine the anti-proliferative effects of EESB in various cancer cell lines such as liver cancer (Hep3B), melanoma (B16F10), leukemia (U937), breast cancer (MDA-MB-231 and MCF-7) and lung cancer (A549) cells, the cells were treated with different concentrations of EESB $(100-500 \mu \mathrm{g} / \mathrm{mL})$ for 24 hours and cell viability was detected by an MTT assay. As shown in Figure 1, the cell viabilities in all the six types of cancer cells were decreased in a concentration-dependent manner. For instance, when U937 cells were treated with $100 \mu \mathrm{g} / \mathrm{mL}$ and $200 \mu \mathrm{g} / \mathrm{mL}$ of EESB, their cell viability decreased by $28 \%$ and $47 \%$, respectively, compared to

A

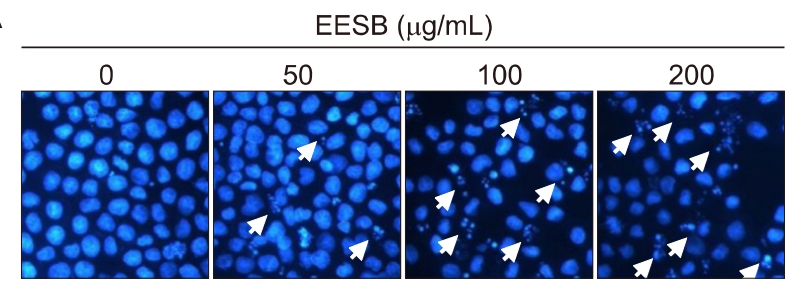

B

B $\operatorname{EESB}(\mu \mathrm{g} / \mathrm{mL})$
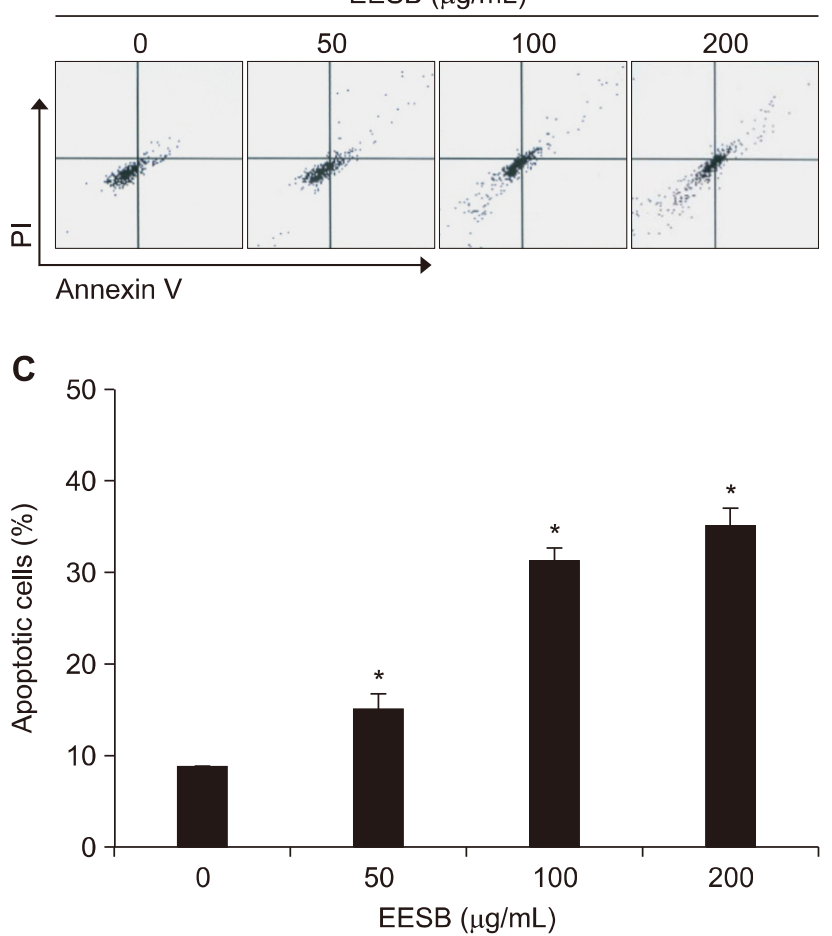

Figure 2. Induction of apoptosis by ethanol extracts of Scutellaria baicalensis roots (EESB) in U937 cells. Cells were plated onto 6-well plates at a density of $4.0 \times 10^{5}$ cells/well and treated with variable concentrations of EESB for 24 hours. (A) Nuclei stained with 4,6-Diamidino-2-phenylindole solution were photographed with a fluorescence microscope using a blue filter $(\times 400)$. Arrows indicate apoptotic cells. (B, C) To quantify the degree of cell apoptosis, the cells were stained with annexin V-FITC and propidium iodide (PI), and the percentage of apoptotic cells (annexin $\mathrm{V}^{+}$cells) was determined using flow cytometric analysis. Data are presented as mean \pm SD values obtained from three independent experiments. ${ }^{*} P<0.05$ compared with the untreated control group. 
the control group. Since U937 cells were more sensitive to EESB than the other cancer cell lines, the subsequent experiments were performed using U937 cells.

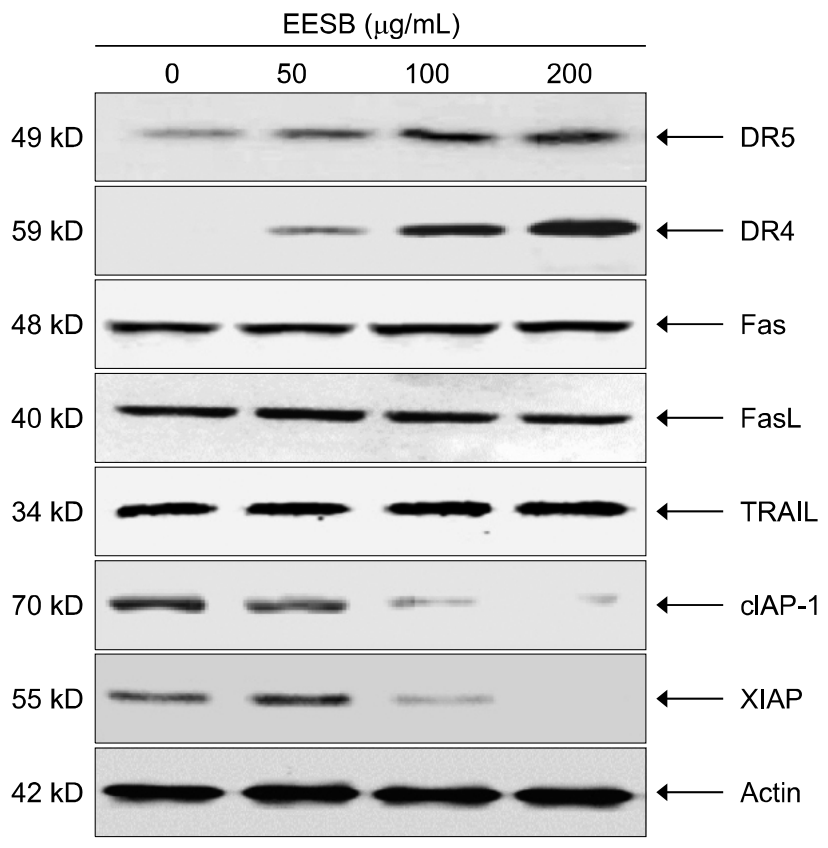

Figure 3. Effects of ethanol extracts of Scutellaria baicalensis roots (EESB) on the expression of death receptor (DR)-related and inhibitor of apoptosis protein (IAP) proteins in U937 cells. Cells grown under the same conditions (shown in Figure 2) were lysed, and cellular proteins were visualized using the indicated antibodies and an enhanced chemiluminescence detection system. Actin was used as an internal control.

\section{Ethanol extracts of $S$. baicalensis roots induce apoptosis in U937 cells}

To determine whether this inhibitory effect of EESB on U937 cell growth was due to apoptotic cell death, we stained the nuclei with DAPI solution. As shown in Figure 2A, the cells treated with EESB exhibited significant chromatin condensation and formation of apoptotic bodies in a concentration-dependent manner. In addition, to determine the degree of apoptosis in the cells treated with EESB, we used flow cytometry. As shown in Figure $2 \mathrm{~B}$ and $2 \mathrm{C}$, the frequency of apoptosis was significantly increased with increasing concentrations of EESB.

\section{Ethanol extracts of $S$. baicalensis roots modulate the expression of apoptosis regulatory genes in U937 cells}

To examine which apoptotic pathway contributes to EESB-induced U937 cell apoptosis, the roles of DRs and their corresponding ligands were examined by Western blot analyses. As indicated in Figure 3, the expression levels of DR4 and DR5 protein were concentration-dependently increased after EESB treatment, but the protein levels of Fas, FasL and TRAIL were not changed. Under the same conditions, EESB treatment resulted in a decrease in the expression levels of the inhibitor of apoptosis protein (IAP) family of proteins, such as CIAP-1 and XIAP.

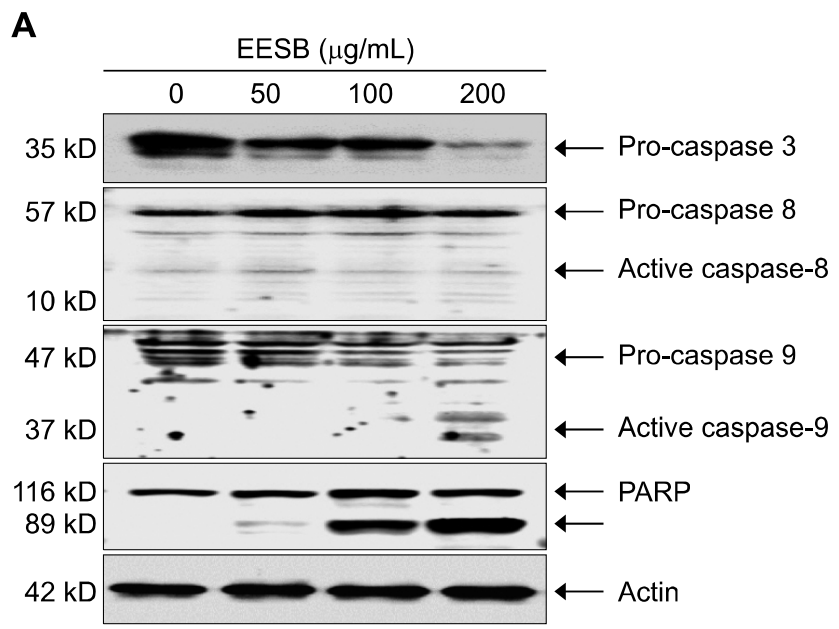

B

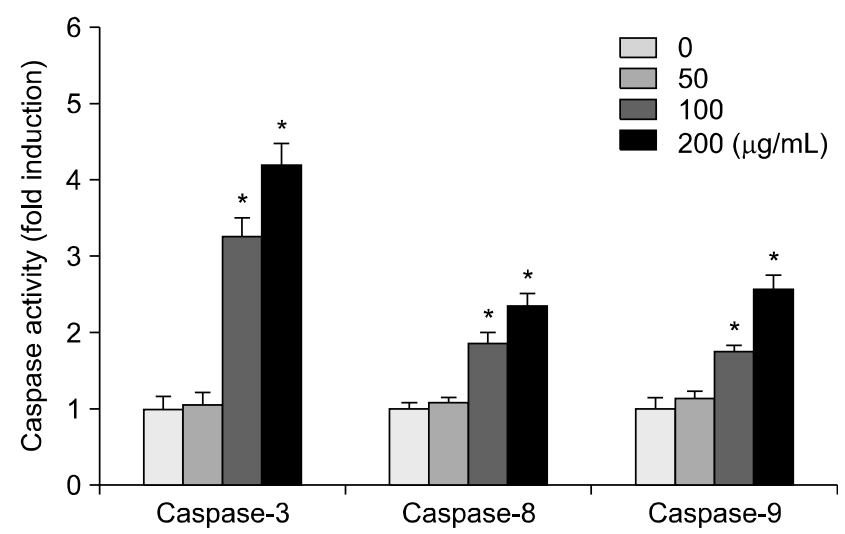

Figure 4. Activation of caspases and degradation of caspase substrates by ethanol extracts of Scutellaria baicalensis roots (EESB) in U937 cells. (A) Cells grown under the same conditions (shown in Figure 2) were lysed, and cellular proteins were visualized using the indicated antibodies and an enhanced chemiluminescence detection system. Actin was used as an internal control. (B) Cells were lysed, and aliquots $(50 \mu \mathrm{g}$ protein) were assayed for in vitro caspase-3, -8, and -9 activity using DEVD-p-nitroaniline (pNA), IETD-pNA, and LEHD-pNA as substrates, respectively, at $37^{\circ} \mathrm{C}$ for 1 hour. The released fluorescent products were analyzed. Data are presented as mean \pm SD values obtained from three independent experiments. $* P<0.05$ compared with the untreated control group. 


\section{Ethanol extracts of $S$. baicalensis roots induce the activation of caspases in U937 cells}

We next examined the expression level and activity of caspases in EESB-induced U937 cells. As shown in Figure 4A, the levels of

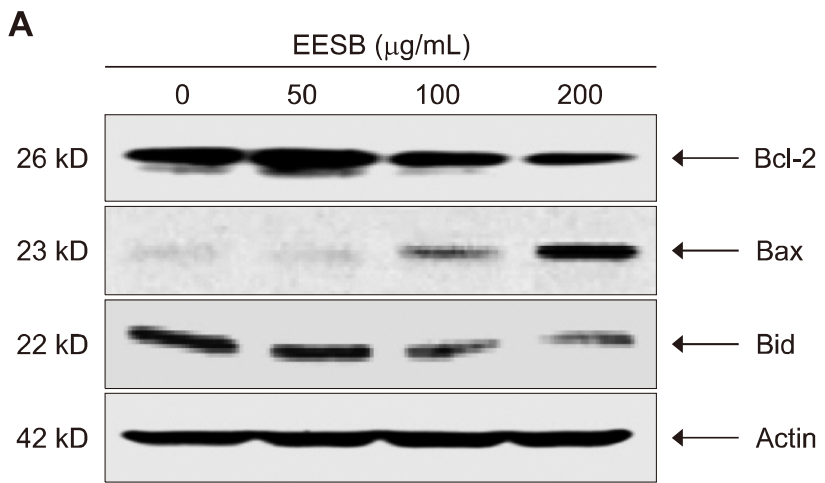

B
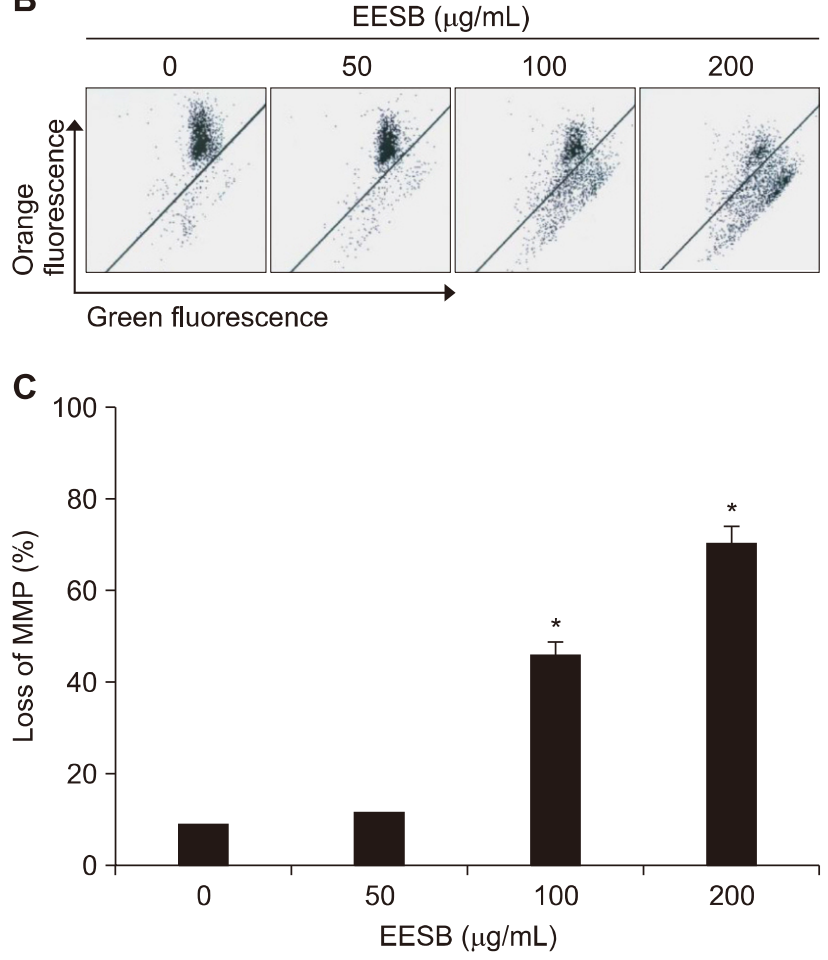

Figure 5. Effects of ethanol extracts of Scutellaria baicalensis roots (EESB) on the expression of Bcl-2 family proteins and values of mitochondrial membrane potential (MMP) in U937 cells. (A) Cells were treated with EESB for 24 hours, and aliquots containing the total protein levels were subjected to SDS PAGE followed by immunoblot analysis with the specific antibodies. (B, C) To quantify the degree of MMP loss, untreated control and EESB-treated cells were incubated with $10 \mu \mathrm{M} \mathrm{JC}-1$ for 20 minutes at $37^{\circ} \mathrm{C}$ in the dark. The cells were then washed once with PBS, and MMP loss was evaluated using a flow cytometer. Data are presented as the mean \pm SD values obtained from three independent experiments. ${ }^{*} P<0.05$ compared with the untreated control group. pro-caspase-3, an effector caspase, in cells treated with EESB were downregulated in a concentration-dependent manner. And, the levels of the active forms of caspase- 8 and caspase-9, initiator caspases of the extrinsic and intrinsic apoptosis pathways, respectively, were markedly increased, and their pro-forms were downregulated. Under the same conditions, the in vitro activities of these caspases were significantly increased in a concentration-dependent manner (Fig. 4B). Moreover, EESB treatment induced progressive proteolytic cleavage of PARP (Fig. $4 A)$, which are substrate proteins of active caspase-3 [25,26].

\section{Ethanol extracts of $S$. baicalensis roots activate the mitochondrial apoptosis pathway in U937 cells}

To confirm induction of EESB-induced apoptotic pathways, the effects of EESB on the levels of Bcl-2 family proteins and MMP values were examined. As indicated in Figure 5A, the levels of the pro-apoptotic protein Bax were upregulated, but the levels of the anti-apoptotic protein $\mathrm{Bcl}-2$ were downregulated in response to EESB treatment. Additionally, although we did not detect the truncated form of the pro-apoptotic protein Bid, a BH3-only protein [27], EESB resulted in a decrease in the intact form of Bid proteins that reflects Bid cleavage and activation (Fig. 5). Moreover, EESB increased the loss of MMP comparison to the untreated control cells (Fig. 5B and 5C).

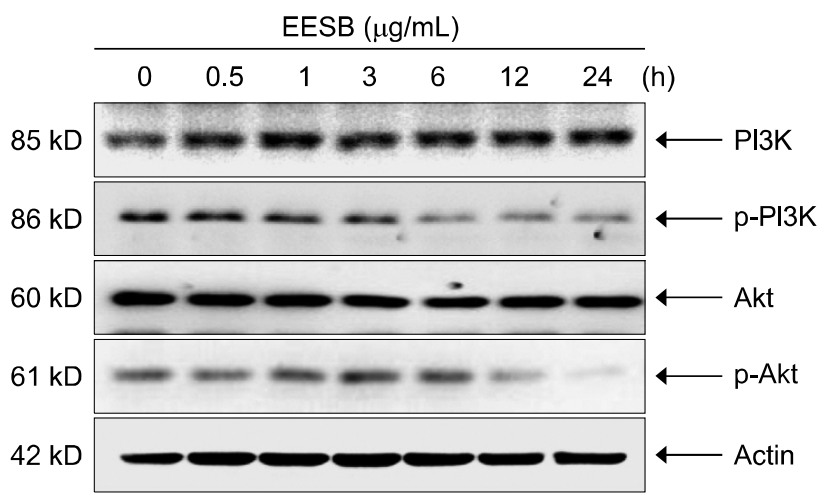

Figure 6. Inactivation of the phosphatidyl inositol 3-kinase (PI3K)/Akt signaling pathway by ethanol extracts of Scutellaria baicalensis roots (EESB) in U937 cells. The cells were treated with EESB $(200 \mathrm{mg} / \mathrm{ml})$ for the indicated times. Equal amounts of cell lysate were resolved on SDS PAGE, transferred to nitrocellulose membranes and probed with the anti-p-PI3K, anti-PI3K, anti-p-Akt and anti-Akt antibodies. The proteins were visualized using an enhanced chemiluminescence detection system. Actin was used as an internal control. 


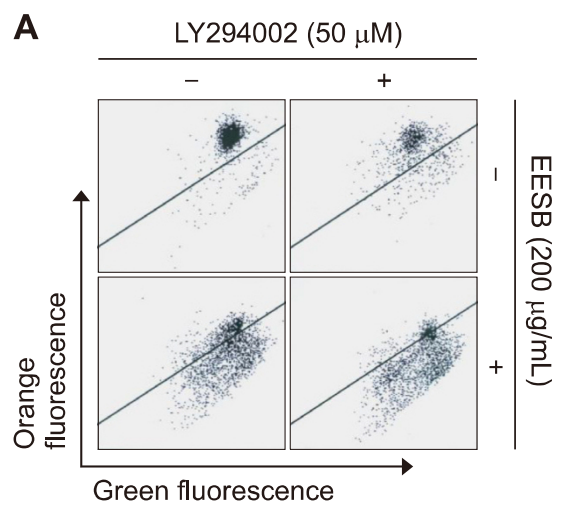

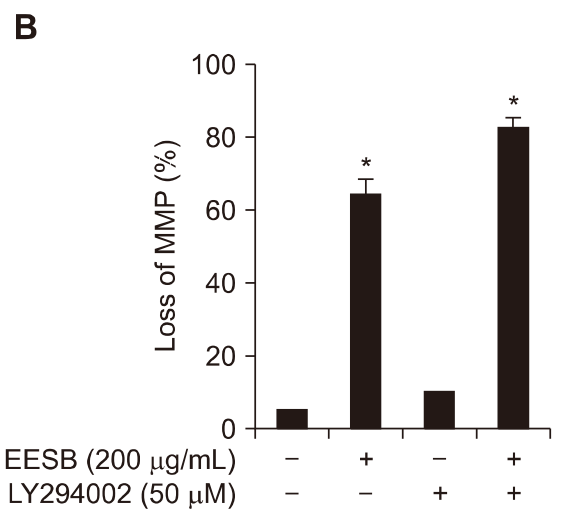

$\mathbf{E}$

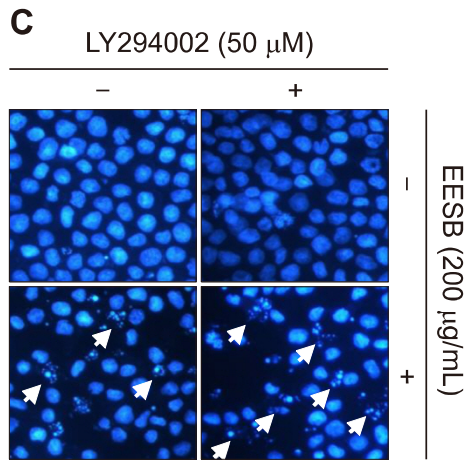

D

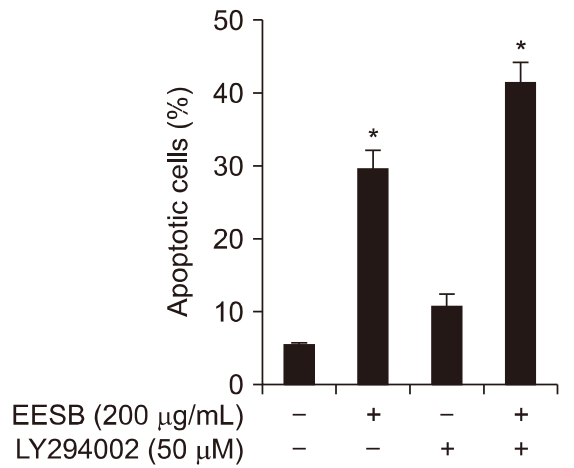

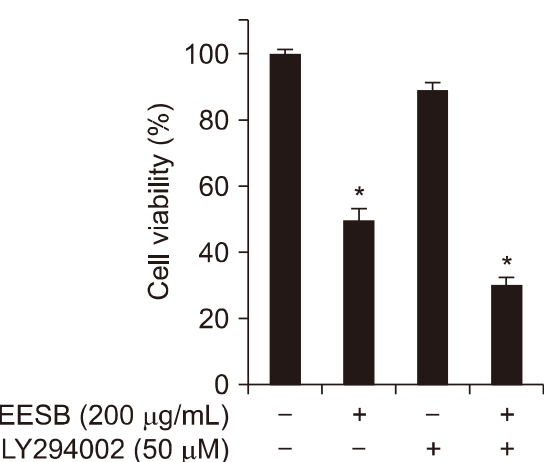

Figure 7. Increase in ethanol extracts of Scutellaria baicalensis roots (EESB)-induced apoptosis by inactivation of phosphatidyl inositol 3-kinase/Akt signaling in U937 cells. The cells were pre-treated with or without LY294002 (50 mM) for 1 hour before treatment with EESB (200 $\mathrm{mM}$ ) for 24 hours. (A, B) Mitochondrial membrane potential (MMP) loss was measured using a flow cytometer. (C) Nuclei stained with 4,6-Diamidino-2-phenylindole solution were photographed with a fluorescence microscope using a blue filter $(\times 400)$. Arrows indicate apoptotic cells. (D) The percentage of apoptotic cells (annexin $\mathrm{V}^{+}$cells) was analyzed using a flow cytometer. (E) Cell viability was determined by an MTT assay. Each point represents the mean \pm SD value of three independent experiments. $* P<0.05$ vs. untreated control cells.

\section{Inactivation of the phosphatidyl inositol 3-kinase/} Akt pathway is involved in ethanol extracts of $S$. baicalensis roots-induced apoptosis in U937 cells

To investigate the relevance of the PI3K/Akt pathway in EESB-induced apoptosis, we evaluated the expression levels of the phosphorylated forms of PI3K and Akt protein, a downstream kinase of PI3K, during EESB-induced apoptosis. Our immunoblotting data showed that phosphorylated PI3K and Akt levels were markedly reduced in response to EESB treatment (Fig. 6). To confirm the relevance of the PI3K/Akt signaling pathway in EESB-induced apoptosis, we examined whether EESB increases or decreases apoptosis in the presence of the representative PI3K/Akt inhibitor LY290042. As shown in Figure 7, when pretreated with LY294002, MMP loss, chromatin condensation, apoptotic body formation and accumulation of annexin V-FITC staining cells increased and cell viability decreased; these effects were greater than those observed with EESB alone.

\section{DISCUSSION}

In the current study, we investigated whether EESB induces apoptosis in human leukemia U937 cells and identified the mechanism involved in apoptosis. Our results clearly demonstrate that EESB inhibits the growth of U937 cells by induction of apoptosis, which seems to explain the anticancer activity. Furthermore, our results show that EESB induces inactivation of the PI3K/Akt signaling pathway.

Apoptotic pathways are broadly divided into extrinsic pathways that require DR-mediated interactions and intrinsic pathways that induce apoptosis through mitochondria-mediated stimulation $[28,29]$. The interaction between DRs and ligands initiates the extrinsic pathway at the cellular membrane, followed by activation of caspase-8, which activates effector caspases such as caspase-3 and -7 [8,30]. Some cells do not die in response to the activation of the extrinsic pathway and require an amplification step induced by caspase- 8 for the activation of the 
intrinsic pathway. In such a situation, caspase- 8 cleaves the $\mathrm{BH}$-only protein $\mathrm{Bid}$ (BH3-interacting-domain death agonist) and converts it to the active form ( $\mathrm{tBid}$ ). $\mathrm{t}$-Bid activates pro-apoptotic multi-domain proteins to increase mitochondrial outer membrane permeability, thus co-engaging the intrinsic pathway $[9,27]$. The damage of the mitochondrial function by a wide range of stimuli can causes the activation of the intrinsic pathway through activation of caspase-9 and, which than activates effector caspases [10,31]. The activation of effector caspases results in the cleavage of several key cellular substrate proteins, including PARP, which acts as a marker of caspase-dependent apoptosis [25,26]. The present data revealed that EESB treatment increased the expression of DR4 and DR5, the crucial proteins of the extrinsic pathway. Under the same conditions, EESB inhibited the expression of IAP family proteins such as XIAP and CIAP-1, which protect against apoptosis due to direct inhibition by the binding of caspases [32-35]. The results also indicated that EESB reduced the levels of pro-caspase- 8 and -9 and enhanced their catalytic activity. Moreover, EESB treatment caused a reduction in the level of total whole Bid proteins, which may be related to the activation of Bid. In addition, EESB markedly activated caspase-3, a key executioner caspase, and led to the cleavage of PARP. Furthermore, EESB-induced apoptosis was associated with downregulation of the anti-apoptotic Bcl-2 protein and loss of MMP in U937 cells. Therefore, the present data indicate that EESB induces Bid truncation by activation of caspase- 8 , leading to the release of pro-apoptotic factors into the cytosol through increase in mitochondrial dysfunction. This data suggests that the activation of both extrinsic and endogenous pathways may contribute at least to the induction of apoptosis by EESB in U937 cells.

Of the various intracellular signaling pathways, the PI3K/Akt signaling pathway plays an important role in regulating cell fate survival in a number of physiological and pathological conditions [36,37]. This pathway is more involved in increasing cell survival through activation of downstream regulators that inhibit apoptosis $[38,39]$. Therefore, a number of studies have shown a close correlation between the inactivation of the PI3K/Akt pathway and the increased cell death probability [29,40]. This indicates that the inactivation of the PI3K/Akt signaling pathway can serve as an effective strategy for the treatment of cancers. Therefore, we further investigated whether inactivation of the PI3K/Akt pathway is associated with EESB-induced U937 cell apoptosis and found that the PI3K/Akt signaling pathway was inactivated in U937 cells exposed to EESB. In addition, when the PI3K/Akt inhibitor, LY294002, was co-treated with EESB, the inhibition of cell viability, loss of MMP and apoptosis were further increased, indicating that PI3K/Akt might play the role of a survival signaling in EESB-induced U937 cell apoptosis.

In summary, the results of the current study demonstrate that EESB induces apoptosis through the activation of the mitochondrial-mediated intrinsic pathway as well as the death-receptor-mediated extrinsic pathway, which is mediated by the PI3K/AKT signaling pathway in human leukemia U937 cells. Although the results of this study may be helpful in understanding the mechanism of EESB's anti-cancer action, further research is needed to determine the relevance of other signaling pathways to the discovery of bioactive agents in EESB.

\section{ACKNOWLEDGMENTS}

The research was supported by the Basic Science Research Program through the National Research Foundation of Korea (NRF) grant funded by the Korea government (2018R1A2 B2005705).

\section{CONFLICTS OF INTEREST}

No potential conflicts of interest were disclosed.

\section{REFERENCES}

1. Adams JM. Ways of dying: multiple pathways to apoptosis. Genes Dev 2003;17:2481-95.

2. Kalimuthu S, Se-Kwon K. Cell survival and apoptosis signaling as therapeutic target for cancer: marine bioactive compounds. Int J Mol Sci 2013;14:2334-54.

3. Wong RS. Apoptosis in cancer: from pathogenesis to treatment. J Exp Clin Cancer Res 2011;30:87.

4. Farkona S, Diamandis EP, Blasutig IM. Cancer immunotherapy: the beginning of the end of cancer? BMC Med 2016;14:73.

5. Assunção Guimarães C, Linden R. Programmed cell deaths. Apoptosis and alternative deathstyles. Eur J Biochem 2004:271: 1638-50.

6. Reed JC. Apoptosis-targeted therapies for cancer. Cancer Cell 2003:3:17-22.

7. Ward TH, Cummings J, Dean E, Greystoke A, Hou JM, Backen A, et al. Biomarkers of apoptosis. Br J Cancer 2008;99:841-6.

8. Sun SY, Hail N Jr, Lotan R. Apoptosis as a novel target for cancer chemoprevention. J Natl Cancer Inst 2004;96:662-72.

9. Hensley P, Mishra M, Kyprianou N. Targeting caspases in cancer therapeutics. Biol Chem 2013;394:831-43.

10. Wang C, Youle RJ. The role of mitochondria in apoptosis. Annu Rev Genet 2009;43:95-118.

11. Avisetti DR, Babu KS, Kalivendi SV. Activation of p38/JNK pathway is responsible for embelin induced apoptosis in lung cancer cells: transitional role of reactive oxygen species. PLoS One 
2014;9:e87050.

12. Bodur C, Kutuk O, Karsli-Uzunbas G, Isimjan TT, Harrison P, Basaga $\mathrm{H}$. Pramanicin analog induces apoptosis in human colon cancer cells: critical roles for Bcl-2, Bim, and p38 MAPK signaling. PLoS One 2013;8:e56369.

13. Gao N, Budhraja A, Cheng S, Liu EH, Chen J, Yang Z, et al. Phenethyl isothiocyanate exhibits antileukemic activity in vitro and in vivo by inactivation of Akt and activation of JNK pathways. Cell Death Dis 2011;2:e140.

14. Li SS, Tang QL, Wang SH, Chen YH, Liu JJ, Yang XM. Simultaneously targeting Bcl-2 and Akt pathways reverses resistance of nasopharyngeal carcinoma to TRAIL synergistically. Tumori 2011;97:762-70.

15. Zhu X, Jiang H, Li J, Xu J, Fei Z. Anticancer effects of Paris saponins by apoptosis and PI3K/AKT pathway in gefitinib-resistant non-small cell lung cancer. Med Sci Monit 2016;22:1435-41.

16. Hong GE, Kim JA, Nagappan A, Yumnam S, Lee HJ, Kim EH, et al. Flavonoids identified from Korean Scutellaria baicalensis Georgi inhibit inflammatory signaling by suppressing activation of NF$\kappa \mathrm{B}$ and MAPK in RAW 264.7 cells. Evid Based Complement Alternat Med 2013;2013:912031.

17. Yimam M, Brownell L, Hodges M, Jia Q. Analgesic effects of a standardized bioflavonoid composition from Scutellaria baicalensis and Acacia catechu. J Diet Suppl 2012;9:155-65.

18. Zhou Y, Yang ZY, Tang RC. Bioactive and UV protective silk materials containing baicalin - the multifunctional plant extract from Scutellaria baicalensis Georgi. Mater Sci Eng C Mater Biol Appl 2016;67:336-44

19. Yoon JJ, Jeong JW, Choi EO, Kim MJ, Hwang-Bo H, Kim HJ, et al. Protective effects of Scutellaria baicalensis Georgi against hydrogen peroxide-induced DNA damage and apoptosis in $\mathrm{HaCaT}$ human skin keratinocytes. EXCLI J 2017;16:426-38.

20. Xu C, Ji GE. Bioconversion of flavones during fermentation in milk containing Scutellaria baicalensis extract by Lactobacillus brevis. J Microbiol Biotechnol 2013;23:1422-7.

21. Jeong K, Shin YC, Park S, Park JS, Kim N, Um JY, et al. Ethanol extract of Scutellaria baicalensis Georgi prevents oxidative damage and neuroinflammation and memorial impairments in artificial senescense mice. J Biomed Sci 2011;18:14.

22. Huang WH, Lee AR, Yang $\mathrm{CH}$. Antioxidative and anti-inflammatory activities of polyhydroxyflavonoids of Scutellaria baicalensis GEORGI. Biosci Biotechnol Biochem 2006;70:2371-80.

23. Pan TL, Wang PW, Leu YL, Wu TH, Wu TS. Inhibitory effects of Scutellaria baicalensis extract on hepatic stellate cells through inducing G2/M cell cycle arrest and activating ERK-dependent apoptosis via Bax and caspase pathway. J Ethnopharmacol 2012; 139:829-37.

24. Park JR, Lee MC, Moon SC, Kim J, Ha KT, Park EJ, et al. Scutellaria baicalensis Georgi induces caspase-dependent apoptosis via mitogen activated protein kinase activation and the generation of reactive oxygen species signaling pathways in MCF-7 breast cancer cells. Mol Med Rep 2017;16:2302-8.

25. Yuan K, Sun Y, Zhou T, McDonald J, Chen Y. PARP-1 regulates resistance of pancreatic cancer to TRAIL therapy. Clin Cancer Res 2013:19:4750-9.
26. Liu F, Jiang N, Xiao ZY, Cheng JP, Mei YZ, Zheng P, et al. Effects of poly (ADP-ribose) polymerase-1 (PARP-1) inhibition on sulfur mustard-induced cutaneous injuries in vitro and in vivo. Peer J 2016;4:e1890.

27. Schneider-Jakob S, Corazza N, Badmann A, Sidler D, Stuber-Roos $\mathrm{R}$, Keogh A, et al. Synergistic induction of cell death in liver tumor cells by TRAIL and chemotherapeutic drugs via the BH3-only proteins Bim and Bid. Cell Death Dis 2010;1:e86.

28. Khaw-on P, Banjerdpongchai R. Induction of intrinsic and extrinsic apoptosis pathways in the human leukemic MOLT-4 cell line by terpinen-4-ol. Asian Pac J Cancer Prev 2012;13:3073-6.

29. Goc A, Kochuparambil ST, Al-Husein B, Al-Azayzih A, Mohammad S, Somanath PR. Simultaneous modulation of the intrinsic and extrinsic pathways by simvastatin in mediating prostate cancer cell apoptosis. BMC Cancer 2012;12:409.

30. Fiandalo MV, Kyprianou N. Caspase control: protagonists of cancer cell apoptosis. Exp Oncol 2012;34:165-75.

31. Miura M. Apoptotic and nonapoptotic caspase functions in animal development. Cold Spring Harb Perspect Biol 2012;4: a008664.

32. Yang L, Mashima T, Sato S, Mochizuki M, Sakamoto H, Yamori T, et al. Predominant suppression of apoptosome by inhibitor of apoptosis protein in non-small cell lung cancer $\mathrm{H} 460$ cells: therapeutic effect of a novel polyarginine-conjugated Smac peptide. Cancer Res 2003;63:831-7.

33. Watson RW, Fitzpatrick JM. Targeting apoptosis in prostate cancer: focus on caspases and inhibitors of apoptosis proteins. BJU Int 2005;96 Suppl 2:S30-4.

34. Keating J, Tsoli M, Hallahan AR, Ingram WJ, Haber M, Ziegler DS. Targeting the inhibitor of apoptosis proteins as a novel therapeutic strategy in medulloblastoma. Mol Cancer Ther 2012;11:2654-63.

35. Do M, Kwak IH, Ahn JH, Lee IJ, Lee JH. Survivin protects fused cancer cells from cell death. BMB Rep 2017;50:361-6.

36. Bhutani J, Sheikh A, Niazi AK. Akt inhibitors: mechanism of action and implications for anticancer therapeutics. Infect Agent Cancer 2013:8:49.

37. Hyun S, Kim MS, Song YS, Bak Y, Ham SY, Lee DH, et al. Peroxisome proliferator-activated receptor-gamma agonist 4-O-methylhonokiol induces apoptosis by triggering the intrinsic apoptosis pathway and inhibiting the PI3K/Akt survival pathway in SiHa human cervical cancer cells. J Microbiol Biotechnol 2015:25:334-42

38. Chen J, Hou R, Zhang X, Ye Y, Wang Y, Tian J. Calycosin suppresses breast cancer cell growth via ER $\beta$-dependent regulation of IGF-1R, p38 MAPK and PI3K/Akt pathways. PLoS One 2014;9:e91245.

39. Kresty LA, Weh KM, Zeyzus-Johns B, Perez LN, Howell AB. Cranberry proanthocyanidins inhibit esophageal adenocarcinoma in vitro and in vivo through pleiotropic cell death induction and PI3K/AKT/mTOR inactivation. Oncotarget 2015;6:33438-55.

40. Hsieh MJ, Tsai TL, Hsieh YS, Wang CJ, Chiou HL. Dioscin-induced autophagy mitigates cell apoptosis through modulation of PI3K/Akt and ERK and JNK signaling pathways in human lung cancer cell lines. Arch Toxicol 2013;87:1927-37. 